\title{
Synthetic Cannabinoids: An Emerging Cause of Coagulopathy
}

\author{
Shakir Ullah ${ }^{\mathrm{a}}$, Amna M. Chaudhary ${ }^{\mathrm{b}}$, Khalid Nawab ${ }^{\mathrm{c}, \mathrm{d}}$, \\ Ravi Athwanic ${ }^{\mathrm{c}}$, Amandeep Singh ${ }^{\mathrm{c}}$
}

\begin{abstract}
Synthetic cannabinoids-associated coagulopathy cases are on the rise but are usually undetected in clinical settings due to less awareness among physicians and the lack of available literature on the subject. Patient may also not volunteer information about the use of such substances therefore detailed history is important. We present a case of a patient found to have coagulopathy of unclear cause as patient did not report the use of synthetic cannabinoid initially.
\end{abstract}

Keywords: Coagulopathy; Synthetic cannabinoids; Marijuana

\section{Introduction}

Synthetic cannabinoids (SC) are a heterogeneous group of compounds used as a substitute for natural marijuana. Its use has increased over the years due to its easy availability and its increasing popularity across the USA. The past decade has seen a nearly 10-fold increase in the availability of highly potent SC for recreational use. These changes have been accompanied by the emergence of serious adverse cardiovascular events, including myocardial infarction, cardiomyopathy, arrhythmias, stroke, and cardiac arrest [1]. SC use leads to increased mental health problems compared with natural cannabinoids [2]. The US Food and Drug Administration (FDA) has already issued a warning regarding the adverse effects of SC [3]. These compounds are associated with several other adverse effects including nausea, vomiting, shortness of breath, hypertension, tachycardia, chest pain, acute renal failure, anxiety, agitation, psychosis, cognitive impairment, hemorrhagic strokes idiopathic Thrombocytopenia Purpura as well as other coagulopathies [4-6].

Manuscript submitted August 4, 2020, accepted August 12, 2020

Published online September 23, 2020

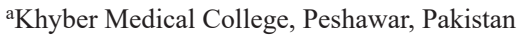

bUPMC Pinnacle Harrisburg Hospital, Harrisburg, PA, USA

${ }^{\mathrm{c}}$ Geisinger Holy Spirit Hospital, Camp Hill, PA, USA

${ }^{\mathrm{d} C}$ Corresponding Author: Khalid Nawab, Geisinger Holy Spirit Hospital, Camp

Hill, PA, USA. Email: khalid.nwb@gmail.com

doi: https://doi.org/10.14740/jmc3561
An increasing trend of coagulopathies in the USA has been reported after the rise of SC use with even some large outbreaks reported over the years [7]. Almost all the cases of $\mathrm{SC}$ induced coagulopathies believed to have occurred because of a compound called brodifacoum [8].

\section{Case Report}

A 47-year-old female with a previous diagnosis of anxiety, depression as well as a history of suicide attempt with an overdose of Benadryl presented to the emergency room (ER) with complaints of right lower quadrant pain, blood in urine and fever, 2 days after a visit to a primary care physician who had prescribed ciprofloxacin for a urinary tract infection (UTI), diagnosed by a urinalysis. Initial blood workup was insignificant with normal levels of hemoglobin, hematocrit, and platelets. Computed tomography (CT) scan of the abdomen and pelvis revealed nonspecific right perinephric and periureteric stranding. She was discharged home with no changes made to medications.

The patient returned to the ER 2 days later with worsening abdominal pain and gross hematuria with the passage of clots. Physical examination revealed an anxious and malnourished female. She was in no distress. Cardiovascular, respiratory, and neurological examinations were unremarkable.

Blood work revealed normal/near normal white blood cells count, hemoglobin, hematocrit, platelets count, normal liver enzymes as well as normal renal function. However, prothrombin time (PT) was greater than $300 \mathrm{~s}$ (normal range is 11 to $13.5 \mathrm{~s}$ ), international normalized ratio (INR) was greater than 25 (normal range is 0.8 to 1.1 ) and activated partial thromboplastin time (aPTT) was $112 \mathrm{~s}$ (normal range is 30 - $40 \mathrm{~s}$ ). Her PT/INR and aPTT values were confirmed three times by laboratory. Further studies revealed normal thrombin time, fibrinogen and factor $\mathrm{V}$ activity. Factor 2 activity was $30 \%$ (normal range for adults is $75-145 \%$ ), factor $\mathrm{X}$ activity $25 \%$ (normal range is $50-200 \%$ ). Plasma coumadin was not detected, urine culture subsequently revealed normal urogenital flora. Blood culture did not show any growth.

Ultrasound of the abdomen revealed large heterogeneous material within the bladder lumen. The patient was admitted to the medical floor and a consult was placed to the department of urology and hematology.

On further workup, we found that the patient had no histo- 
ry of blood transfusions or spontaneous bleeding. The patient denied a history of familial bleeding disorders. The patient had not been prescribed warfarin or any novel oral anticoagulants (NOAC) and denied taking any other anticoagulants. The patient admitted to smoking half a pack of cigarettes a day and smoked marijuana for 21 years as well as drank alcohol daily. The patient was initially not very forthcoming with her history of alcohol and drug abuse and the history was obtained on multiple, separate occasions. She subsequently admitted to using synthetic marijuana recently.

She was evaluated by the department of hematology for coagulopathy. The case was discussed with poison control due to concerns for synthetic marijuana-induced coagulopathy. The patient was started on scheduled vitamin $\mathrm{K}$ which was gradually increased until her INR was less than 2.0. She was discharged home with oral vitamin K $15 \mathrm{mg}$ every $6 \mathrm{~h}$ and advised to follow-up with the hematology office and get INR checked before the appointment.

\section{Discussion}

$\mathrm{SC}$ use is on the rise over the past years due to their perceived safety as compared to natural marijuana as well as their ease of availability [9]. It cannot be detected using routine drug test and thus is popular among the newer generation [10]. Over the years, there has been an increasing trend in ER visits due to $\mathrm{SC}$, with a $900 \%$ increase reported by a study conducted in Maryland with 40 cases in 2013 to 353 in 2017, with the majority of the patients being females [11].

The Illinois poison center reported 178 cases between March 10 and August 1, 2018, with suspected SC use and coagulopathy. Eighty-eight percent of those had an INR greater than 10. Eighteen patients underwent qualitative anticoagulant testing, with all of them reporting positive for brodifacoum. $63 \%$ of the patient in the study presented with flank-pains and $70 \%$ had hematuria [7]. In a study conducted by Castaneto et al about $92 \%$ of respondent admitted to taking SC with other drugs and/or alcohol [5].

An outbreak of SC-associated coagulopathy reported in Illinois showed that the causative agents were brodifacoum, difenacoum, and bromadiolone, which are potent, long-acting anticoagulant rodenticides (LAAR) [12]. But brodifacoum is by far the biggest culprit in SC-associated coagulopathies. All reported cases of cannabinoid-associated coagulopathies where vitamin $\mathrm{K}$ antagonists were suspected had identified contamination with brodifacoum [13].

Enhanced toxicity of SC compared to marijuana is due to the high affinity of these compounds for cannabinoid receptor type 1 (CB1) and cannabinoid receptor type 2 (CB2) as compared to tetrahydrocannabinol (THC), the active compound present in marijuana [14]. Brodifacoum is a 4-hydroxycoumarin anticoagulant characterized as a "super warfarin" anticoagulant [15]. Brodifacoum inhibits the enzyme vitamin $\mathrm{K}$ epoxide reductase, steadily decreasing the level of active vitamin $\mathrm{K}$ in the blood, which is required for the synthesis of important substances including prothrombin involved in blood clotting. This disruption leads to blood effectively losing any ability to clot [2].

In addition to coagulopathy, $\mathrm{SC}$ use has also led to acute kidney injury (AKI) with or without other associated systems. A study conducted after Wyoming Department of Health found AKI in 16 patients all of whom reported use of SC [16].

Imaging can be performed to evaluate the presence of bleeding, especially in patients with severe abdominal or flank pain. With most common radiographic abnormalities were in the renal system, as seen on computed tomography, including perinephric stranding, hyperemia, diffuse thickening, and dilatation of the urothelial collecting system [17].

Specific guidelines for treatment have not been established yet but treatment often requires intravenous vitamin K1 at the dose of 50 to $100 \mathrm{mg}$; due to long half-life difenacoum, chronic maintenance with $100 \mathrm{mg}$ per os (PO) vitamin K1 daily may be used to suppress coagulopathy. In previously reported cases, treatment courses averaged 168 days. In case of active bleeding, hemostatic therapy with recombinant factor VIIa and prothrombin complex concentrate has been reported, and phenobarbital has been used to expedite LAAR metabolism [18].

\section{Conclusions}

Patients presenting with unexplained coagulopathy should be questioned about the use of SC as patients may not be forthcoming with such information due to various reasons. It may be associated with AKI and can be treated with vitamin K supplementation however guidelines regarding treatment of such coagulopathy have not been established yet.

\section{Acknowledgments}

None to declare.

\section{Financial Disclosure}

No funding was received. None of the authors have disclosures relevant to this manuscript.

\section{Conflict of Interest}

None to declare.

\section{Informed Consent}

Informed consent was obtained from the patient. The manuscript has been sufficiently de-identified to protect the patient.

\section{Author Contributions}

All authors contributed to the editing of the manuscript. SU, 
AMC and $\mathrm{KN}$ wrote the manuscript. RV and $\mathrm{AS}$ reviewed and made final additions.

\section{Data Availability}

The authors declare that data supporting the findings of this study are available within the article.

\section{References}

1. Pacher P, Steffens S, Hasko G, Schindler TH, Kunos G. Cardiovascular effects of marijuana and synthetic cannabinoids: the good, the bad, and the ugly. Nat Rev Cardiol. 2018;15(3):151-166.

2. Mensen VT, Vreeker A, Nordgren J, Atkinson A, de la Torre R, Farre M, Ramaekers JG, et al. Psychopathological symptoms associated with synthetic cannabinoid use: a comparison with natural cannabis. Psychopharmacology (Berl). 2019;236(9):2677-2685.

3. Statement from FDA warning about significant health risks of contaminated illegal synthetic cannabinoid products that are being encountered by FDA | FDA. https:// www.fda.gov/news-events/press-announcements/statement-fda-warning-about-significant-health-risks-contaminated-illegal-synthetic-cannabinoid. Accessed July 27, 2020.

4. Castaneira G, Rojas K, Galili Y, Field Z, Perez-Perez A, Madruga M, Carlan SJ. Idiopathic thrombocytopenic purpura induced by synthetic cannabinoid. J Addict Med. 2019;13(3):235-236.

5. Castaneto MS, Gorelick DA, Desrosiers NA, Hartman RL, Pirard S, Huestis MA. Synthetic cannabinoids: epidemiology, pharmacodynamics, and clinical implications. Drug Alcohol Depend. 2014;144:12-41.

6. Rose DZ, Guerrero WR, Mokin MV, Gooch CL, Bozeman AC, Pearson JM, Burgin WS. Hemorrhagic stroke following use of the synthetic marijuana "spice". Neurology. 2015;85(13):1177-1179.

7. Devgun JM, Rasin A, Kim T, Mycyk MB, Bryant SM, Wahl MS, DesLauriers C, et al. An outbreak of severe coagulopathy from synthetic cannabinoids tainted with
Long-Acting anticoagulant rodenticides. Clin Toxicol (Phila). 2020;58(8):821-828.

8. Panigrahi B, Jones BC, Rowe SP. Brodifacoum-contaminated synthetic marijuana: clinical and radiologic manifestations of a public health outbreak causing life-threatening coagulopathy. Emerg Radiol. 2018;25(6):715-718.

9. Springer YP, Gerona R, Scheunemann E, Shafer SL, Lin $\mathrm{T}$, Banister SD, Cooper MP, et al. Increase in adverse reactions associated with use of synthetic cannabinoids - Anchorage, Alaska, 2015-2016. MMWR Morb Mortal Wkly Rep. 2016;65(40):1108-1111.

10. Panlilio LV, Goldberg SR, Justinova Z. Cannabinoid abuse and addiction: clinical and preclinical findings. Clin Pharmacol Ther. 2015;97(6):616-627.

11. Atanda A, Goodell JC, Adams S, Black V. Synthetic cannabinoid-related ED visit trends in Maryland using ESSENCE, 2013-2018. Online Journal of Public Health Informatics. 2019;11(1).

12. Tole M, LaBedz S, Feinstein DL, Rubinstein I. Adherence to long-term follow-up of patients with life-threatening, inhaled synthetic cannabinoids-associated coagulopathy in Chicago. Lung. 2019;197(3):349-352.

13. Arepally GM, Ortel TL. Bad weed: synthetic cannabinoid-associated coagulopathy. Blood. 2019;133(9):902905.

14. Tai S, Fantegrossi WE. Pharmacological and toxicological effects of synthetic cannabinoids and their metabolites. Curr Top Behav Neurosci. 2017;32:249-262.

15. Patocka J, Petroianu G, Kuca K. Toxic potential of superwarfarin: brodifacoum. Military Medical Science Letters. 2013;82(1):32-38.

16. Acute kidney injury associated with synthetic cannabinoid use - Multiple States. 2012. https:/www.cdc.gov/ mmwr/preview/mmwrhtml/mm6206a1.htm. Accessed July 27, 2020.

17. Kelkar AH, Smith NA, Martial A, Moole H, Tarantino MD, Roberts JC. An outbreak of synthetic cannabinoid-associated coagulopathy in Illinois. N Engl J Med. 2018;379(13):1216-1223.

18. King N, Tran MH. Long-acting anticoagulant rodenticide (Superwarfarin) poisoning: a review of its historical development, epidemiology, and clinical management. Transfus Med Rev. 2015;29(4):250-258. 